\title{
EFECTO DE TRES ESTRATEGIAS DE ENSEÑANZA CENTRADAS \\ EN PROCESOS DE PENSAMIENTO SOBRE EL RENDIMIENTO ESTUDIANTIL EN ESTEQUIOMETRÍA ${ }^{1}$
}

\section{EFFECT OF THREE LEARNING STRATEGIES FOCUSED ON} PROCESSES OF THOUGHT ON STUDENTS' ACHIEVEMENT

\section{Ana Yarihtza Alvarado *}

UPEL-IPB

Recibido 17-03-08

\section{RESUMEN}

La presente investigación realizada bajo un diseño cuasi experimental, permitió determinar el efecto del Tasier, el Mapa de Conto dete y la Resoluc de Problemas como estragias de enseñza centadas Problemas como estrategias de enseñanza centradas en procesos de pensamiento sobre el Rendimiento Estudiantil en Estequiometría. Para ello se aplicó un Prueba de Conocimientos Previos (PCP) antes de abordaje del contenido a 114 alumnos, seleccionados como muestra del primer año de ciencias de Educación Media Diversificada y Profesional de la UEN Rafael Villavicencio. Luego de los tratamientos experimentales se les administró una post prueba con el fin de determinar el aprendizaje alcanzado en Estequiometría por dichos alumnos. Los resultados obtenidos permitieron concluir a través de un análisis de varianza, que el uso de cualquiera de las tres estraias que estrategias antes referidas, ayudará en igual medida a estudiante a construir su propio conocimiento y desarrollar habilidades y destrezas cognitivas, permitiéndole así obtener un mejor desenvolvimiento en sus estudios.

Descriptores: estrategias de ensenanza, rendimiento estudiantil, procesos de pensamiento

\footnotetext{
Trabajo presentado como requisito parcial para optar al Grado de Magíster en Educación Mención Enseñanza de la Química. UPEL-IPB

** Profesora en Ciencias Naturales Mención Química (UPEL-IPB); Magíster en Enseñanza de la Química (UPEL-IPB); Diplomados en Investigación (IUETAEB) y en Formación de Tutores de Trabajos y Tesis de Grado (UPEL-IPB), Dip C Do Experimental Libertador (UPEL-IPB). anayarihtza@egmail.com
}

\section{INTRODUCCIÓN}

Las reformas y transformaciones del currículo en el sector educativo venezolano, específicamente a nivel de educación básica, generaron a su vez reformas en el nivel universitario, particularmente en aquellas instituciones que administran la formación docente. Tal es el caso de la Universidad Pedagógica Experimental Libertador (UPEL), donde todos los elementos que conforman la actividad educativa como docente, alumno, medio y currículo se han evaluado y han sido objetos de estudio tanto en pregrado como en postgrado. No escapando a esto, la especialidad de Química que desde 1997 contempla, en su diseño curricular del componente de práctica profesional, la producción y aplicación de estrategias de enseñanza y aprendizaje; así el futuro especialista llevará a cabo su labor atendiendo a dos aspectos relevantes: el familiarizar al estudiante con las tareas del quehacer científico y el de incrementar su nivel de desarrollo mental. En relación a este último y a la edad en que se encuentran los estudiantes del noveno grado de educación básica, Piaget (citado por Astofi, 2001) señala que el individuo se encuentra en la etapa de operaciones formales y por lo tanto puede resolver problemas abstractos, utilizar la lógica, poseer un pensamiento más científico y desarrollar temas de interés.

Sin embargo, investigaciones realizadas por especialistas en materia del pensamiento, opiniones de docentes universitarios especialistas en el área de Química, así como eventos académicos e instituciones relacionadas con la asignatura, conforman una realidad académica que contrasta con lo anteriormente planteado. Es así como el VII encuentro de la Escuela para la Enseñanza de la Química (2004) por ejemplo, se planteó que existen muchas dificultades en el proceso de enseñanza y aprendizaje de la Química, específicamente en los contenidos inherentes a la Estequiometría, lo que incide negativamente en la capacidad del alumno para enfrentar exitosamente estudios universitarios o para transferir los conocimientos básicos obtenidos de esta área, a problemas de su entorno.

Sánchez (1998) por su parte reportó, que los estudiantes del primer año de Educación Superior presentan limitaciones en su capacidad cognitiva para procesar información, debido a que los docentes no buscan estimular dichos procesos cognitivos.

Según la autora citada, el problema que existe en cuanto a la enseñanza y aprendizaje de algunas asignaturas como la Química, dado lo abstracto de la mayoría de sus contenidos, induce al docente a revisar su práctica instruccional y evaluar su pertinencia a favor del desarrollo del pensamiento formal del individuo. 
Desde estas perspectivas el sistema educativo debe adaptarse a los nuevos paradigmas de aprendizaje, motivo por el cual la presente investigación aborda un tema que reviste interés para una comunidad docente que constantemente exige metodologías que le ayuden a superar las dificultades cotidianas del aula, relacionadas con los contenidos abstractos de la asignatura, en momentos en que se da la construcción del conocimiento y se imprime una gran importancia al cómo aprende el individuo y cómo debe enseñársele a aprender. Vale la pena insistir en la importancia que tiene enseñarles a los alumnos estrategias que le permitan mejorar su comprensión, organizar y controlar su propio aprendizaje, dado que es imperativo que el alumno desarrolle destrezas en el manejo de instrumentos, aprenda a tabular datos, procese información y la transfiera procesada a su quehacer diario, motivo por el cual la presente investigación pretendió dar respuesta a la siguiente interrogante.

¿Qué efecto tendría sobre el aprendizaje de la estequiometría la aplicación de tres estrategias de enseñanza centradas en procesos de pensamiento?

Sobre la base de esta interrogante, se presentó un trabajo de investigación para evaluar el efecto de la aplicación del Taller, el Mapa de Concepto y la Resolución de Problemas como estrategias de enseñanza centradas en procesos del pensamiento, sobre el aprendizaje del contenido de Estequiometría. Debido a que según Sánchez (2002), la metodología de enseñanza y aprendizaje centrada en procesos del pensamiento, permite al estudiante adquirir aprendizajes significativos y aplicables en la búsqueda de soluciones a los problemas propios de su entorno. Además el nivel de Educación Media Diversificada y Profesional debe preparar al estudiante en los conocimientos, habilidades, destrezas y valores que le capaciten para continuar con su formación a nivel universitario.

Lo expuesto anteriormente demuestra la importancia que tiene el uso de estrategias centradas en procesos de pensamiento para el aprendizaje significativo de los contenidos de Química. De allí que el presente artìculo se exponen los resultado de un trabajo de grado que tuvo como objetivo ddeterminar el efecto del Taller, el Mapa de Concepto y la Resolución de Problemas como Eestrategias de Enseñanza Centradas en Procesos de Pensamiento sobre el Rendimiento Estudiantil de los estudiantes del primer año de ciencias en la UEN Rafael Villavicencio de Barquisimeto en el Aprendizaje de la Estequiometría.

\section{ALGUNAS CONSIDERACIONES TEÓRICAS}

El presente estudio se sustenta en las teorías del aprendizaje significativo de Ausubel y el modelo constructivista de Vygotski. Asimismo, la fundamentación teórica se apoya en información relativa a didáctica centrada en procesos, estrategias de enseñanza y aprendizaje, estrategias de enseñanza centradas en procesos de pensamiento, Taller, Mapa de Concepto, Resolución de Problemas, aprendizaje de contenido y Estequiometría.

\section{Constructivismo}

La concepción psicológica constructivista se concibe, según Díaz y Hernández (2003), como un movimiento pedagógico que considera al aprendiz como un ente activo, capaz de construir su conocimiento, sobre la base de sus potencialidades y experiencias, en conjunción con el contexto ambiental que lo rodea.

De acuerdo con la teoría constructivista, las personas aprenden modificando viejas ideas en lugar de acumular porciones nuevas, por lo tanto, una efectiva estrategia de enseñanza facilita el proceso constructivo y conduce a una autonomía creciente.

Por otra parte, los autores antes citados, refieren que el constructivismo es un movimiento pedagógico que se presenta en las modalidades de: (a) constructivismo genético, (b) constructivismo disciplinario y (c) constructivismo social. Donde el máximo representante del constructivismo genético es Jean Piaget (ob. cit.), quien indica que los factores con mayor relevancia en la construcción del conocimiento son la genética y las experiencias que pueda tener el aprendiz con el medio ambiente.

Según, Ausubel, Novak y Gowin en el constructivismo disciplinario, el énfasis se centra en la metodología, para facilitar la enseñanza y el aprendizaje.

Para efectos de este estudio abordaremos el constructivismo social fundamentado en la teoría de Vygotskiana, porque plantea que el conocimiento es una función de cómo el individuo crea significados a partir de sus propias experiencias y que el verdadero aprendizaje es una construcción que logra modificar la estructura mental de cada alumno, dado que, el verdadero conocimiento es el resultado de una elaboración o construcción personal, producto de un proceso interno de pensamiento en el curso del cual el sujeto coordina entre sí diferentes nociones, les atribuye un significado, las organiza y las relaciona con otros anteriores.

\section{Aprendizaje Significativo}

El Aprendizaje significativo es, obviamente, aprendizaje con significado. Es significativo por cuanto el aprendiz es capaz de explicar o resolver problemas nuevos, en pocas palabras los nuevos conocimientos (conceptos, ideas, proposiciones, modelos, fórmulas) pasan a significar algo para él. Para eso, en el aula, el aprendiz debe presentar una predisposición para aprender y los materiales educativos deben ser potencialmente 
significativos. Sin embargo, tales condiciones son necesarias, pero no suficientes; el significado está en las personas, no en las cosas o eventos.

El aprendizaje significativo implica una relación indisociable de aprendizaje y desarrollo, por cuanto ayuda a clarificar los procesos de construcción genética del conocimiento (Ausubel, citado en UPEL, 1998). Según este autor, los nuevos significados no son las ideas o contenidos, objetivos presentados y ofrecidos al aprendizaje, sino que son el producto de un intercambio, de una fusión. Los nuevos significados son generados por la interacción de la nueva idea o concepto potencialmente significativo con las ideas pertinentes que ya posee el alumno en su estructura cognitiva. En otras palabras, para que el alumno aprenda de manera significativa, deberá relacionar de manera no-arbitraria y noliteral los significados previos con aquellos que captó de los materiales potencialmente significativos del currículum. Pero en ese proceso, tanto el profesor como el alumno deben tener y desarrollar una conciencia semántica, de tal manera que el aprendizaje pueda ser significativo y crítico.

Por ello, en el proceso de aprendizaje significativo se debe comenzar por conocer la peculiar estructura mental de los individuos, antes de asignar las tareas de aprendizaje.

Lo anteriormente planteado deja entrever la importancia que tiene para el docente apoyarse en una teoría de aprendizaje y su correspondiente teoría instruccional, sin menospreciar el contenido de la materia que va a enseñar.

\section{Didáctica Centrada en Procesos}

Enseñar al alumno a aprender además de ser un objetivo relevante, es un medio para alcanzar los demás objetivos educativos, donde las capacidades de autorregulación de los alumnos y la metacognición desempeñan un papel fundamental. Barrero, citado en Carranza y Celaya (2003).

Además, las destrezas metacognitivas son especialmente importantes en el aprendizaje de las Ciencias, desde el momento que el alumno toma conocimiento de sus propias capacidades cognitivas y sobre la forma de regularlas. Vargas y Arbeláez, citados en Carranza y Celaya ( Ob.cit. ). No obstante, sería absurdo hablar con certeza de enseñanza si previamente no aclaramos cómo aprende el que aprende y cuáles son los procesos que hacen posible dicho aprendizaje.

Según Ruíz, C. (1998), el sistema educativo ha enfocado de tres maneras distinta el desarrollo cognoscitivo del niño: (a) A través del supuesto implícito de desarrollar la mente mediante el estudio intensivo de determinadas disciplinas (v.g. latín, matemática, lógica); (b) Utilizando programas específicamente diseñados para enseñar a pensar; y (c) Entrenando la habilidad de pensar, a través de la utilización del contenido del plan de estudio.

En consonancia con la opinión de numerosos investigadores el abordaje del último enfoque es muy importante, debido a que no requiere de ningún programa e intervención especial sino, más bien, de un cambio en la estrategia de enseñanza por parte del docente en el proceso de aprendizaje.

La didáctica basada o centrada en procesos pensamiento es una forma instruccional que combina el desarrollo de los contenidos curriculares con el entrenamiento de los procesos psico-afectivos del estudiante. En esta modalidad los contenidos cumplen un fin impuesto por el currículo y son utilizados por el docente con el deliberado propósito de desarrollar en el alumno los procesos internos que hacen posible el aprendizaje.

\section{Estrategias de Enseñanza y Aprendizaje}

El Ministerio de Educación, en el Manual del Docente para la Educación Básica (1997), define a las estrategias de enseñanza y aprendizaje como el conjunto concatenado de métodos, técnicas y recursos que se planifican y organizan, considerando las diversas situaciones instruccionales orientadas a facilitar el proceso de enseñanza y aprendizaje.

Para efectos de este estudio, se entiende como estrategia de enseñanza y aprendizaje el desarrollo de una acción educativa efectiva que evita las improvisaciones, facilite la distribución de los contenidos e incluya recursos, métodos y técnicas, de acuerdo con la realidad social y pedagógica del educando.

\section{Estrategias de Enseñanza Centradas en Procesos de Pensamiento}

Según Flores (2000), las estrategias centradas en procesos de pensamiento son aquellas que están orientadas a favorecer la construcción del conocimiento con la participación dinámica y activa del alumno. Entre las estrategias centradas en procesos de pensamiento destacan el taller, el mapa de concepto y resolución de problemas, el trabajo en equipo y los juegos didácticos (Sánchez, 2003).

Para efectos del presente estudio se seleccionaron las estrategias Taller, Mapa de Concepto y Resolución de Problemas, las cuales se describen a continuación. 


\section{El Taller como Estrategia de Enseñanza y Aprendizaje}

El Taller es una forma de enseñar y sobre todo, de aprender mediante la realización de algo que se lleva a cabo conjuntamente entre educando y educador.

Según la Universidad Nacional Abierta (UNA, 2003), el Taller se fundamenta en una relación directa entre participantes y facilitador, cuyo objetivo básico es lograr la integración de los basamentos teóricos con la ejecución práctica. Desde el punto de vista pedagógico, se apoya en determinados supuestos y principios. Ander-Egg (1990), destaca ocho clasificaciones fundamentales para caracterizar el Taller en cuanto a modelo de enseñanza y aprendizaje.

1. Es un aprender haciendo mediante la integración y globalización de la formación teórica con la práctica, otorgando prioridad a la apropiación del saber a través de la acción/reflexión.

2. Es una metodología participativa se enseña y se aprende a través de una experiencia en común en la que todos están implicados e involucrados como sujetos/agentes.

3. Es una pedagogía de la pregunta contrapuesta a la pedagogía de la respuesta propia de la educación tradicional, el conocimiento se produce fundamentalmente y casi exclusivamente en respuestas a preguntas, buscando respuestas, sin instalarse nunca en certezas absolutas.

4. Es un entrenamiento que tiende al trabajo interdisciplinario y enfoque sistemático, es un esfuerzo por conocer y operar, asumiendo el carácter multifacético y complejo de la realidad.

5. La relación docente-alumno queda establecida en torno a la realización de una tarea común porque se crean las condiciones pedagógicas y de organización para que los naturales protagonistas del proceso de educación (educando y educadores) puedan decidir acerca de la marcha de dicho proceso.

6. Posee carácter globalizante e integrador en su práctica pedagógica porque consiste en adquirir el conocimiento de un tema desde múltiples perspectivas, dado que la realidad nunca se presenta fragmentada de acuerdo con la clasificación de las ciencias o la división de las disciplinas académicas.

7. Implica y exige un trabajo grupal, y el uso de técnicas adecuadas, dado que el taller es un grupo social organizado para el aprendizaje y como tal alcanzará una mayor productividad y gratificación grupal se usan técnicas adecuadas. Como en toda forma pedagógica, el proceso de aprendizaje es personal, el trabajo grupal no excluye actividades y tareas individuales, por el contrario exige una complementariedad entre lo individual y lo grupal.

8. Permite integrar en un sólo proceso, tres instancias, como son la docencia, la investigación y la práctica, lo sustancial es realizar un proyecto en el que docente y alumno participen activa y responsablemente. La docencia se ejerce a partir de la situación de enseñanza/aprendizaje, a través de la reflexión teórica sobre la acción; la investigación es la exigencia previa a la acción y la práctica son las actividades y tareas que se llevan a cabo en la realización del proyecto.

Según Ander Egg (Ob.cit) el uso del taller como estrategia de enseñanza debe hacernos reflexionar acerca de que no todo lo que se hace con este nombre en sentido estricto, ni todo lo que se hace a través de él, supone una transformación pedagógica significativa por si sola. Debemos considerar la necesidad de investigar acerca del taller e interesarnos por los integrantes que lo hacen posible, por la autoridad, responsabilidad y liderazgo del docente dentro del grupo y muy especialmente por el intercambio de vivencias entre ellos. De igual manera debemos considerar que la sobreabundancia de palabras por parte del docente puede limitar la capacidad de la búsqueda formal del conocimiento por parte del alumno.

Por otro lado, Troielli, D. (2001) cree que los talleres deben desarrollarse en un ambiente grato y relajado, respetando los tiempos de aprendizaje, fomentando el compañerismo y la comunicación entre sus participantes.

De lo anteriormente expuesto podemos deducir la importancia de incorporar en nuestras clases, el taller como estrategia intelectual acorde a los intereses de los alumnos para satisfacer así sus inquietudes y expectativas.

\section{El Mapa de Concepto como Estrategia de Enseñanza y Aprendizaje}

El Mapa de Concepto, según Novak (1998), representa un resumen esquemático de lo aprendido y ordenado de una manera jerárquica. En él, el conocimiento se presenta organizado y representado en niveles de abstracción, situando los más generales e inclusivos en la parte superior y los más específicos y menos inclusivos en la parte inferior.

El Mapa de Concepto representa un diagrama en el cual se muestran las relaciones existentes entre los conceptos en forma de proposiciones., según Ontoria (ob. cit.) permite:

1. Conectar contenidos cognitivos con contenidos conceptuales considerando las ideas previas. 
2. Orienta hacia la búsqueda de información.

3. Permite reflexionar sobre los mecanismos propios del aprendizaje.

4. Compartir y discernir los conceptos aprendidos.

5. Evaluar antes, durante y después del proceso del aprendizaje detectando aciertos y desaciertos.

En conclusión, los mapas conceptuales representan las relaciones significativas entre conceptos en forma de proposiciones o frases simplificadas: dos o más conceptos ligados por palabras para formar una unidad semántica. Su teoría está basada en un modelo constructivista de los procesos cognitivos humanos, por lo que son ideales para que el estudiante reflexione sobre los cambios ocurridos en su proceso de aprendizaje y los relacione con los conocimientos adquiridos.

\section{La Resolución de Problemas como Estrategia de Enseñanza y}

\section{Aprendizaje}

La enseñanza de las ciencias y en especial de la Química, tal como lo señala Galvis (1993) se relaciona íntimamente con la Resolución de Problemas.

Según Polya (1986), la Resolución de Problemas aparte de enseñar sistemática y activamente problemas cuantitativos, permite resolver los problemas del entorno, mediante la transferencia del conocimiento. Dicha metodología consiste en la aplicación y concientización, por parte del alumno, de cuatro pasos fundamentales que son la comprensión del problema, concepción de un plan de resolución, ejecución del plan elaborado, evaluación o visión retrospectiva.

1. La comprensión del problema consiste en ver claramente lo que se pide. El individuo debe separar las principales partes del problema, la incógnita, los datos y las condiciones.

2. La concepción de un plan se refiere a captar las relaciones que existen entre los diversos elementos.

3. La ejecución del plan consiste en el desarrollo de las actividades a seguir. En ella se cuida cada detalle hasta asegurarse que el razonamiento esté correctamente presentado.

4. La visión retrospectiva es volver atrás. Una vez encontrada la solución, revisarla y discutirla, se consideran los resultados y el camino que condujo a ellos.
En el estudio de la Química, la Resolución de Problemas es de gran importancia porque requiere de la participación activa del alumno y del desarrollo de los procesos básicos del pensamiento. Esto le garantiza además, el reconocimiento y razonamiento lógico de los problemas.

\section{Aprendizaje de Contenidos}

Los bloques de contenido que se enseñan en los currículos se refieren a los conocimientos fundamentales de una disciplina en particular. Estos contenidos pueden agruparse en tres áreas básicas: (a) contenidos declarativos, (b) contenidos procedimentales y (c) contenidos actitudinales. (Díaz y Hernández, ob. cit.).

El conocimiento conceptual es construido a partir del aprendizaje de conceptos, principios y explicaciones, los cuales no tienen que ser aprendidos en forma literal, sino abstrayendo su significado esencial o identificando las características definitorias o reglas que lo componen. Ocurre por una asimilación significativa de la información nueva, se comprende lo que está aprendiendo, pero para eso es imprescindible el uso de conocimientos previos pertinentes que posea el alumno.

Los contenidos procedimentales, según estos autores, se refieren a la ejecución de procedimientos, estrategias, técnicas, habilidades, destrezas y métodos. Es un conocimiento de tipo práctico que incluye la realización de acciones y operaciones.

Y los contenidos actitudinales, son las actitudes y valores a desarrollar en el aprendiz, basándose en dos pilares fundamentales: el desarrollo del individuo (el ser) y el desarrollo del individuo dentro de la sociedad (el convivir).

\section{Aprendizaje del Contenido de Estequiometría}

La Estequiometría estudia las relaciones cuantitativas entre los elementos y compuestos cuando experimentan un cambio químico (Caballero y Ramos, 2002). En términos químicos, la Estequiometría se refiere a los cálculos que se hacen sobre la base de las relaciones de las sustancias que participan en una reacción y que se expresan en una ecuación química, lo cual involucra a la vez cálculos con fórmulas.

\section{DESCRIPCIÒN METODOLÒGICA}

El estudio realizado responde a una investigación de campo, dado que los datos requeridos se obtuvieron directamente de los sujetos participantes. El trabajo se realizó sobre la base de un diseño cuasi-experimental con post prueba, con el propósito de estudiar 
el efecto de la aplicación de tres estrategias centradas en procesos del pensamiento sobre el aprendizaje de la Estequiometría, contenido correspondiente al programa de articulación para el Primer Año de Ciencias de Educación Media Diversificada y Profesional. Ejecutado durante el período escolar 2004-2005, en la UEN Rafael Villavicencio del Distrito Escolar II de la ciudad de Barquisimeto.

\section{Población y Muestra}

La muestra estuvo conformada por 114 estudiantes, tomada de una población de 418 alumnos cursantes de la asignatura Química durante el año escolar 2004-2005, equivalentes en cuanto a edad, sexo y repitencia.

\section{Instrumentos}

En el desarrollo de esta investigación se utilizaron dos instrumentos. El primero fue una Prueba de Conocimientos Previos (PCP) que se les aplicó a los estudiantes antes del tratamiento experimental, con la finalidad de determinar el nivel de dominio que poseían sobre los contenidos programáticos necesarios para comprender el nuevo contenido (Estequiometría). El segundo denominado Prueba de Aprendizaje sobre el Contenido de Estequiometría (PASCE), se les aplicó después del tratamiento a los mismos grupos experimentales (Taller, Mapa Conceptual y Resolución de Problemas) bajo la figura de post prueba con la finalidad de medir el nivel de aprendizaje adquirido por estos en Estequiometría, durante el desarrollo del procedimiento experimental.

\section{Variables del Estudio}

\section{Variable Independiente}

En este estudio se trabajó, como variable independiente, la estrategia de enseñanza y aprendizaje, definida conceptualmente según el Manual del Docente para la Educación Básica (1987), como el conjunto de métodos, técnicas y recursos que se planifican de acuerdo con las necesidades de la población a la cual va dirigida, los objetivos que se persiguen y la naturaleza de las áreas y asignaturas; todo esto con la finalidad de hacer más efectivo el proceso de enseñanza y aprendizaje.

\section{Cuadro 1}

Definición operacional de las dimensiones de la Variable Independiente

\begin{tabular}{|c|c|c|}
\hline Taller & Mapa de Concepto & $\begin{array}{l}\text { Resolución de } \\
\text { Problemas }\end{array}$ \\
\hline $\begin{array}{l}\text { Modalidad utilizada por el docente } \\
\text { para abordar el contenido de Estequiometría y } \\
\text { desarmollar procesos del pensamiento en los } \\
\text { alumnos, es medido a través de los siguientes } \\
\text { indicadores: } \\
\text { * Los alumnos agrupados en equipo } \\
\text { construyen una definición de Estequiometría } \\
\text { a través de un organizador de ideas. } \\
\text { * En sesión plenaria el alumno } \\
\text { expone al grupo las ideas o conclusiones de } \\
\text { su equipo de trabajo. } \\
\text { * Cada equipo de trabajo analiza la } \\
\text { guía didáctica y realiza los ejercicios } \\
\text { propuestos para luego explicarlos al resto del } \\
\text { grupo. } \\
\text { * Los alumnos agrupados en pareja } \\
\text { deberán analizar el contenido de reactivos } \\
\text { limitante y relacionarlo con los problemas } \\
\text { sencillos de su entomo. }\end{array}$ & $\begin{array}{l}\text { Medio utilizado por el docente para } \\
\text { ayudar a los alumnos a captar los contenidos } \\
\text { objeto de estudio y a desarrollar las habilidades } \\
\text { cognitivas, medido a través de los siguientes } \\
\text { indicadores: } \\
\text { * El alumno identifica la idea } \\
\text { principal a partir de un texto dado. } \\
\text { * Ordena y jerarquiza las palabras } \\
\text { claves o conceptos. } \\
\text { * Establece relaciones entre las ideas } \\
\text { mediante el uso de líneas. } \\
* \text { Describe la relación entre las ideas. } \\
\text { * Establece significados de la } \\
\text { relación entre las ideas. } \\
\text { * Construye Mapas de Conceptos } \\
\text { acerca del contenido de Estequiometría a } \\
\text { través del análisis de partes. }\end{array}$ & $\begin{array}{l}\text { Medio utilizado por } \\
\text { el docente para que el alumno } \\
\text { interprete, planifique, ejecute } \\
\text { y evalúe el contenido de } \\
\text { Estequiometría utilizando } \\
\text { para ello los procesos del } \\
\text { pensamiento. }\end{array}$ \\
\hline
\end{tabular}

\section{Variable Dependiente}

La variable dependiente rendimiento estudiantil, se define según Gaceta Oficial de la República Bolivariana de Venezuela $N^{\circ}$ 36.787, decreto $N^{\circ} 313$ del 15 de Septiembre de 1999, en su artículo 16, como el progreso alcanzado por los alumnos en función de las competencias, bloques de contenido y objetivos programáticos propuestos, fundamentado en el conocimiento de los contenidos curriculares y ciertas destrezas cognitivas.

Operacionalmente, rendimiento estudiantil es el promedio de calificaciones que se obtuvo de la aplicación de una prueba sobre el contenido de Estequiometría correspondiente a la I unidad del Programa de Articulación del Primer Año de Ciencias de Educación Media Diversificada y Profesional, para la asignatura de Química, en el período escolar $2004-2005$.

\section{LOS RESULTADOS}


Con el propósito de dar respuesta a los objetivos del estudio y en concordancia con las variables e hipótesis de la investigación, se realizó un análisis estadístico a través del programa SPSS a los datos obtenidos tanto en la Prueba de Conocimientos Previos (PCP) como en la Post Prueba, obteniendo como resultado lo siguiente.

Antes del tratamiento, los tres grupos se sometieron a la Prueba de Homogeneidad de la Varianza de Levene, no encontrando diferencias estadísticamente significativas entre los grupos tratados con las estrategias Taller, Mapa Conceptual y Resolución de Problemas.

De igual manera, a los resultados obtenidos en la Prueba de Conocimiento Previos se les hizo un análisis de Varianza (ANOVA), permitiendo demostrar que los grupos estudiados eran grupos equivalentes en cuanto a conocimientos previos.

Seguidamente a través del Coeficiente Estandarizado de Asimetría se determinó que los datos recolectados poseían un comportamiento estadísticamente normal, dado que los valores obtenidos se encontraban dentro del rango de -2 a +2 , tal como lo indica el siguiente cuadro.

\section{Cuadro 2}

\section{Coeficiente Estandarizado de Asimetría}

\begin{tabular}{|c|c|c|c|}
\hline Prueba & $\mathrm{GE}_{1}$ & $\mathrm{GE}_{2}$ & $\mathrm{GE}_{3}$ \\
\hline $\begin{array}{c}\text { Coeficiente de } \\
\text { Asimetría }\end{array}$ & $-0,41$ & 0,38 & 0,97 \\
\hline
\end{tabular}

Nota: $\mathrm{GE}_{1}$ : Estrategia Taller, $\mathrm{GE}_{2}$ : Estrategia Mapa Conceptual, $\mathrm{GE}_{3}$ : Estrategia

\section{Resolución de Problema}

Igualmente, a los resultados de la Post prueba se les aplicó un Análisis de Varianza obteniendo como resultado un valor de $\mathrm{F}$ igual a 1,95 dentro de un rango para el $\mathrm{F}$ crítico de 0,05 y 2,83. Finalmente como $F_{c}>F$, se concluye que no existen diferencias estadísticamente significativas entre los tres grupos experimentales. Por lo que el Taller, el Mapa de Concepto y la Resolución de Problemas como estrategias centradas en procesos de pensamiento son igualmente efectivas en el proceso de aprendizaje del contenido de Estequiometría. Considerando que el nivel de abstracción de la Estequiometría como contenido programático desarrollado durante la investigación es alto y que no se hizo uso del grupo control como tradicionalmente se ve en los diseños cuasi experimentales.

\section{CONCLUSIONES Y RECOMENDACIONES}

Con base en los objetivos propuestos, las hipótesis formuladas y el análisis estadístico de los resultados obtenidos, se plantearon las siguientes conclusiones y recomendaciones:

Al comparar los promedios de las calificaciones obtenidas en la prueba sobre el contenido de Estequiometría la evidencia estadística permitió concluir, que las estrategias Taller, Mapa de Concepto y Resolución de Problemas, como estrategias centradas en procesos de pensamiento utilizadas en esta investigación para el estudio del contenido programático antes referido, fue estadísticamente no diferenciado en los tres grupos de estudiantes sometidos al tratamiento experimental. Por lo que se puede afirmar, que el uso de cualquiera de estas tres estrategias ayudará en igual medida al estudiante a construir su propio conocimiento, activando así los procesos de pensamiento que le van a permitir desarrollar habilidades y destrezas cognitivas. Además, la información obtenida en esta investigación nos permitió estimar el impacto del uso de las estrategias centradas en procesos de pensamiento en una población estudiantil determinada. En consecuencia, estos resultados tienen implicaciones importantes en la práctica pedagógica, porque sirven de referencia al docente de aula, además permiten ampliar el conocimiento que se tiene en cuanto al efecto de las estrategias centradas en procesos de pensamiento sobre el aprendizaje de los contenidos, destacando la importancia de los procesos cognitivos en el aprendizaje. Por lo que se sugiere: Incluir en el currículo educativo su enseñanza y aplicabilidad, dada la transdisciplinariedad del conocimiento.

Realizar investigaciones similares que permitan determinar: (a) si los docentes enseñan los contenidos de Química centrados en procesos del pensamiento y (b) si los estudiantes han desarrollado algunas habilidades cognitivas específicas para el estudio de la Química.

Finalmente, se sugiere evaluar los resultados obtenidos de la puesta en práctica de los ejes transversales por parte de los docentes del nivel de Educación Básica, específicamente el referido al desarrollo del pensamiento.

\section{REFERENCIAS}

Ander-Egg, E. (1999). El taller: una alternativa de renovación pedagógica. Argentina: Editorial Magisterio del Río de la Plata.

Aprile, M. (1998). Efecto de la metodología de resolución de problemas cuantitativos sobre el rendimiento académico la atracción hacia la química y el potencial creativo de los alumnos de química. Trabajo de Maestría. UPEL. Barquisimeto. 
Astolfi, J. (2001). El construccionismo según Piaget. 1era edición. Sevilla, España: Diada editora S.L.

Carranza, M. y Celaya, G. (2003).Una estrategia para favorecer la comprensión y el aprendizaje en las Ciencias Morfológicas: Revista ELectrónica de Investigación y Evaluación Educativa, v. 9, n. 2.

Díaz, F y Hernández, G. (2003). Estrategias docentes para un aprendizaje significativo. Una interpretación constructiva. México: Mc Graw Hill Interamericana Editores, S. A de C. V.

Flores, J. (2002). Química. Noveno grado. Caracas: Editorial Santillana.

Florez, R. (2002). Currículo y Pedagogía. Conferencia Central presentada en V Reunión de Currículo. Caracas.

Graterol, J. L. (2001). Resolución de problemas como estrategia de enseñanza centrada en procesos cognoscitivos y su efecto en la capacidad de análisis y en el conocimiento en la asignatura ciencias biológicas. Trabajo de grado. Barquisimeto.

VI Escuela Venezolana para la Enseñanza de la Química (2004). La Hechicera, Facultad de Ciencias, Universidad de Los Andes, Mérida.

Ministerio de Educación (1997). Manual del Docente para la Educación Básica. Caracas.

Novak, J. (1998). Conocimiento y aprendizaje. Los mapas conceptuales como herramienta facilitadora para escuelas y empresas. Madrid: Alianza.

Ontoria, A. (2001). Mapas conceptuales. Una técnica para aprender. Madrid: Narcea S.A. de Ediciones.

Polya, G. (1986). Cómo plantear y resolver problemas. México: Trillas.

Reglamento General de la Ley Orgánica de Educación (1999). Gaceta Oficial de la República de Venezuela $N^{\circ}$ 36787. Caracas: Imprenta Nacional.

Ruiz, B. C. (1988). La estrategia didáctica mediadora: Una alternativa para el desarrollo de procesos en el aula. Investigación y Postgrado , 3 (2), 57-73.

Sánchez, M. (1998). Proyecto aprender a pensar. Caracas, Venezuela.

Sánchez, M. de (2003). Desarrollo de habilidades del pensamiento (Procesos Básicos del Pensamiento). Bogotá: Norma.
Suárez, F. (1992). Influencias de las ilustraciones en la retención de información de memoria a corto y largo plazo. Caracas.

Universidad Nacional Abierta. Vicerrectorado Académico (Enero,2003). Efectividad en el Manejo de Estrategias y Recursos para la instrucción.

Universidad Pedagógica Experimental Libertador (1998). Praxis constructiva para la aplicación del currículo básico nacional. Convenio Interinstitucional UPEL-IPB / UCER- Lara / FUNDAUPEL.

Universidad Pedagógica Experimental Libertador. Vicerrectorado de Investigación y Postgrado. (2003). Manual de trabajo de grado de especialización y maestría y tesis doctorales. Caracas, Venezuela: Fedupel.

Vigotsky, L. (1979). El desarrollo de los procesos psicológicos superiores. Barcelona, España: Editorial Crítica Grijalbo Mondorori, S.A.

Vigotsky, F. (1993). Pensamiento y lenguaje. Buenos Aires: La Pléyade. 